1-2000 solution of quinine and quinidine base, and incubated at $37^{\circ} \mathrm{C}$. for 24 hours. The solutions were without a trace of hæmolysis.

(1) Quinine bisulphate. (2) Quinine base.

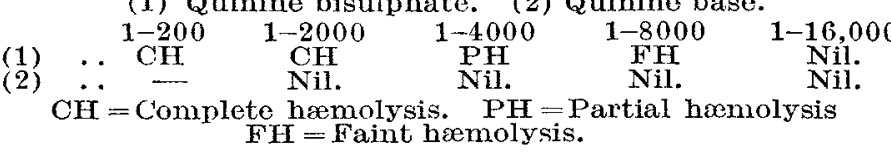

In some experiments done for me by Major H. C. Brown, C.T.E., of the Wellcome Laboratory, it seemed that the base of quinine and quinidine slightly hindered the action of anti-sheep amboceptor when acting on goat's corpuscles. The injection of strong acid solution into the blood causes a considerable amount of hæmolysis, and there can be little doubt that it is the cause of the rigors so commonly seen a few hours after these intravenous injections; $0.1 \mathrm{~g}$. of anhydrous base is dissolved in $29 \mathrm{c.cm}$. of $\mathrm{N} / \mathrm{HCl}$ and made up to about $150 \mathrm{c.cm}$. with distilled water. The acid is then exactly neutralised with $29 \mathrm{c.cm}$. of $\mathrm{N} / \mathrm{NaOH}$, and made up to $200 \mathrm{c.cm}$. This solution is then a 1-2000 solution of base in normal saline, the sodium chloride being formed by the interaction of the $\mathrm{HCl}$ and $\mathrm{NaOH}$. This solution can be boiled without the quinine being converted into quinotoxin, as occurs when heating acid solutions of this alkaloid. A much smaller dose of anhydrous base $0.25 \mathrm{~g}$. injected in this manner will probably be as effective as $0.5 \mathrm{~g}$. of bihydrochloride. As quinine base is not hæmoBytic, the adrocates of the quinine-blackwater theory will now have to turn their attention to the various decomposition products of this alkaloid.

Effect on Blood Pressure.-The fall of blood pressure that occurs after the intravenous injection of quinine is well known, and has been the subject of numerous papers. As I have been advocating the use of quinidine in benign tertian infections, it was necessary to know what effect this alkaloid would have in producing a fall of blood pressure. Dr. H. H. Dale, F.R.S., kindly tested the effect of these various alkaloids for me on the blood pressure of cats. The following are the main conclusions: 1 . The depressor effect was greater in all the "cinchonine series" (most, marked in quinidine), and took longer to wear off than the cinchonidine series. 2. The depressor effect produced by the injection of $1 \mathrm{mg}$. of quinidine was equal to that produced by $10 \mathrm{mg}$. of quinine. Therefore this will be a danger in the intravenous administration of quinidine in the young, feeble, or in Taro doses.

The graph shows the difference in the fall of blood pressure produced by the intravenous injection of $10 \mathrm{mg}$. of hydroquinine and hydroquinidine. The action on uterine muscle is included under the systemic effects for convenience. The action of these alkaloids was tested on the excised guinea-pig's uterus. The following are the main conclusions :-

On Virgin Uterus.-(1) In weak solutions $(1-75,000)$ all the cinchona alkaloids increase the amplitude of the uterine contractions. In stronger concentrations $(1-20,000)$ they depress both the amplitude and rhythm. (2) The cinchonine series cause a greater effect on the amplitude and rhythm than the cinchonidine series. (3) The hydro-alkaloids are more powerful in this respect than the natural alkaloids.

On Multiparous Vterus. The behaviour is very similar to that above, but the muscle is more sensitive and irregular in its action to these stimuli.

On Pregnant Uterus.-I am dealing with the effects of quinine in a separate paper, but the main conclusions were :(1) That the action of quinine depends upon the concentration of the base; thus with strong concentrations (1-40,000) the uterus is thrown into a high state of tonus; with weaker concentrations $(1-100,000)$ the intermittent uterine contractions are increased; and with still weaker concentrations (180,000 and under) have no effect on the contractions. (2) That quinine causes contraction of both the longitudinal and circular fibres.

Miscarriage will therefore only be caused $(a)$ by large doses, almost sufficient to poison the patient, when the uterus will be thrown into a persistent state of tonus which would lead to death of the foetus or rupture of the membranes; (b) by large doses (20-40 gr.), increasing the force of the intermittent uterine contractions, to cause rupture of weak membranes or dilatation of an already open or patulous os. Ordinary medicinal doses, 5-10 gr., in a healthy woman would have no effect on the uterine contractions, as we already know from clinical experience.
Factors on which the Pharmacological Action of These Alkaloids Depend.- $\Lambda$ s far as can be reasoned from this study, the pharmacological action of these cinchona alkaloids is dependent on three factors in the complex alkaloidal molecule:-

1. The grouping of the quinuclidine system around the asymmetrical carbon atom at (3) as shown by their optical rotation. The dextrorotatory alkaloids, "cinchonine series," are more powerful in their effects in toxicity on mice and paramecium, on inhibiting the action of enzymes, on blood pressure and uterine muscle than the lævorotatory alkaloids of the "cinchonidine series." The cinchonidine series are more powerful in their local anæsthetic effects.

2. The vinyl group (CH: $\mathbf{C H}_{2}$ ) in the quinuclidine system. The natural alkaloids are slightly more toxic to paramecium than the hydro-alkaloids. The hydrogenation of the vinyl group to $\mathbf{C H}_{2} \cdot \mathrm{CH}_{3}$ in the hydro-alkaloids renders these alkaloids more difficult to oxidise, and they are accordingly not so easily broken down by the body tissues. They are more toxic to mice, inhibit enzyme action, and cause a greater fall of blood pressure and uterine contraction than the natural alkaloids.

3. The group occupying position (6) in the quinoline ring. The higher members of both series of the hydroalkaloids are more toxic to mice, paramecium, bacteria, and leucocytes, and are more powerful as local anæsthetics. This action is not maintained on blood pressure, uterine muscle, and inhibition of enzyme action.

We can therefore conclude that (1) The dextrorotatory alkaloids, "cinchonine series," are more powerful than their lavorotatory isomerides, the "cinchonidine series" ; (2) the hydro-alkaloids are more stable and more active in many respects than the natural alkaloids; (3) the higher members of both series of these alkaloids are more toxic to mammals, protozoa, and bacteria than the lower members.

These results are the effects produced on the various living tissues, and give the direction in which future research should follow. The parasiticidal action of these alkaloids on the malarial parasite can only be arrived at by direct observations on malarial patients.

\section{A CASE OF LATERAL SINUS THROMIBOSIS.}

\section{BY LESLIE B. C. TROTTER, M.A., M.D. CAMB.}

THrs case of lateral sinus thrombosis, probably due to acute pyorrhoea and alveolar abscess, presents points that seem to me to be of sufficient interest to be placed on record. The patient recovered.

IV. P., was a well-built farm labourer, aged 35. For some days before the onset of any symptoms he had been busy hay-making in a very hot sun. On June 27 th he began to complain of severe headache and giddiness, associated with pain in and in front of the right ear. On the 28th, when he was first seen, there was some swelling in front of the ear with redness and tenderness, but nothing abnormal behind the ear and no otorrhoa. The teeth were all more or less decayed, and the gums were swollen, red, and purulent Temp. $100^{\circ} \mathrm{F}$., pulse 120 per minute. Hot boric fomentations were prescribed, a purge, rest in bed, and a milk diet.

Patient was next seen in response to an urgent message on June 30 th at 4 P.Mr. The swelling in front of the ear had subsided ; up to 1 A.M. he had complained of violent headache and sweating, and at that time he had a severe rigor; he then suddenly felt better and slept heavily for 12 hours. On waking up he had another rigor and vomited just before he was seen the second time. His state on the latter occasion was: Temp. $102^{\circ}$, pulse 120 , acute generalised headache, no pain or tenderness over the mastoid, and no eversion of the ear; very slight discharge from the ear. The swelling in front of the ear had subsided, but there was some slight tenderness behind the angle of the jaw (right side). The face was slightly cyanosed.

Diagnosis. - In view of the extreme heat of the weather there was always the possibility of sunstroke, but the otorrhoea, preauricular swelling, tenderness behind the angle of the jaw, and cyanosis had to be accounted for. A diagnosis therefore was made of "acute otitis media (due to oral sepsis) and lateral sinus thrombosis."

Patient was at once sent to the Cottage Hospital, Ledbury. On July 1st he felt and appeared much better in the morning and it was decided to postpone operation. In the evening he complained of headache, and had a temperature of $99^{\circ}$. On the 2 nd in the morning patient had a rigor and vomited. Temp. $100 \cdot 6^{\circ}$, pulse 88 . In view of developments further delay was decided against, and arrangements were made for operation. 
Operation.-After a hypodermic injection of $1 / 100 \mathrm{gr}$. of atropine, patient was anosthetised by Dr. T. A. Jones with C.E.2 mixture. First step : An incision was made about $4 \frac{1}{2}$. long over the anterior border of the sterno-mastoid on the right side, and the internal jugular vein (which was quite empty) tied with a double ligature of stout catgut below the common facial junction, opposite the bifurcation of the common carotid. A gauze wick 1 in. wide was inserted down to the vein, and the incision sutured (save for $\frac{1}{2}$ in. at the lower end) with silkworm gut. A sterile gauze dressing was then applied. Second step: A curved incision was made behind the car and the mastoid antrum opened. There was no sign of pus, so the bone was partly chiselled and partly gouged away down to the lateral sinus. Directly this was reached a quantity of very foul, blackish liquid pus welled out. The opening in the bone was sufficiently enlarged to admit the little finger-end and the bone bevelled away to ensure free drainage. A quantity of breaking-down blood clot was removed from the lateral sinus, and the cavity washed out with hydrogen peroxide. In view of the patient's grave condition the middle ear was not touched. A drainage-tube was inserted into the lateral sinus, and the wound lightly packed with iodoform gauze. A plain sterile gauze dressing was then applied. Total duration of operation was $1 \frac{1}{2}$ hours.

Progress.-Patient's condition steadily improved. On the 5 th he was put out on the balcony and remained there night and day. The discharge from the wound was extremely foul for a long time in spite of frequent dressings. From the 10th to the 14th the temperature continued to fall, being subnormal all the time. At the same time patient complained of persistent bad headache (generalised over the top of the head) and vomited practically everything. Feeding became difficult; 1 drachm doses of bicarbonate of soda with minim doses of dilute hydrocyanic acid 4-hourly did not have much effect. He was then given $\frac{1}{2}$ oz. doses of champagne every hour and the vomiting ceased in a few hours. On the evening of the $15 \mathrm{th}$, in view of acute headache and a temperature of $102^{\circ}$, associated with a pulse-rate of only 118, an exploring needle attached to a small glass syringe was inserted into the posterior fossa through the inner wall of the lateral sinus, the needle being directed downwards, backwards, and inwards. About 1 c.cm. of turbid serum was withdrawn. No pus was found. A quarter of an hour afterwards the headache ceased abruptly.

On the 14th, 24 hours before the posterior fossa was explored, patient began to be troubled with a copious discharge of pus in the naso-pharynx, which interfered seriously with deglutition, but cleared up in about a week under treatment with a nasal douche and gargle composed of a weak alkaline antiseptic. On the morning of the 16th an incision was made in the wall of the lateral sinus with a fine scalpel on the site of the needle puncture. This opening was kept patent with a gauze drain. The relief of pressure in the posterior fossa on the night of July 15th and the incision of the dura next day marked the turning-point. From this time onwards patient progressed steadily Temperature dropped to normal or subnormal, all vomiting and headache ceased, and he began to take solid food in satisfactory quantities. On August 16th patient had recovered sufficiently to have his teeth dealt with; they were all removed by Mr. A. Moore. Thereafter the cleaning up of the posterior fossa and lateral sinus was remarkably rapid. Patient was discharged from hospital on the 29 th and by the 31 st the wound had completely healed. He has no vertigo or headache, and feels fit for work in spite of a very slight discharge from the ear every night.

There seems to be no doubt that the origin of the sepsis was an acute exacerbation of pyorrhoea due primarily to carious teeth. A septic phlebitis was probably started in the pharyngeal veins, which in turn gave rise to the inflammatory swelling in the parotid region before causing trouble in the internal jugular. The phlebitis in the jugular was followed by an ascending thrombosis, which later involved the lateral sinus. It seems quite likely that the slight discharge from the meatus seen on June 30th came from the parotid swelling direct, and not at that time from the middle ear. The opening of the common facial vein must have been occluded by clot in the jugular, as the latter was quite empty at the site of ligation. The purulent discharge in the naso-pharynx probably arose from an abscess of similar origini.e., septic phlebitis, which burst spontaneously. The middle ear and the posterior fossa no doubt became infected later by direct continuity from the lateral sinus.

My thanks are due to Mr. Wilfred Trotter for his helpful advice, to my partner Dr. Jones, and to Miss Holford, the matron of the Ledbury Cottage Hospital, for her highly efficient care of the patient.

\section{Aftedical Socretires.}

\section{ROYAL SOCIETY OF MEDICINE.}

\section{SECTION OF OPHTHALMOLOGY.}

A MeEtring of this section was held on Jan. 13th, Dr. JAMEs 'TAYLoR, the President, in the chair.

\section{Exhibition of Cases and Specimens.}

Mr. P. G. Doyne showed for diagnosis a female child aged 4 months exhibiting swellings, to the outer side of the limbus in each eye, which had been present since birth. Other congenital defects exhibited by the child were a bifid tongue and three phalanges in the right thumb. The diagnosis lay between dermoid and subconjunctival lipoma. In the discussion which followed the opinion was generally expressed that the swellings were probably dermoids. It was suggested that $\mathrm{X}$ ray photographs should be taken to determine the position of the epiphyses.

\section{Mr. M. S. Mayou exhibited a case of} Osteomyelitis of the Upper Jau

in a child who, at the age of 6 months, suffered from an extremely painful swelling of the eyelids attributed to an insect bite. An incision was made by the family practitioner parallel to the lower margin of the orbit and a quantity of pus evacuated. Some days later the child was brought to hospital, where it was treated for two months, and was finally discharged with the sinus unhealed. A piece of bone had exfoliated recently, and it was loped that the sinus would now heal. A second case, also an infant, was admitted to the same hospital with extreme swelling of the right eye, especially of the lids, which were red and cedematous and very tender. What could be seen of the eyeball on separating the lids was apparently normal, although the eyeball as a whole was thrust forward. The child was extremely ill. ternperature $104^{\circ} \mathrm{F}$. and pulse 140 . Several days before admission an abscess in the hard palate had been incised by the general practitioner, but this had healed completely at the time of admission. An incision parallel to the lower border of the orbit carried down to bone liberated a quantity of pus. An immediate improvement occurred in the child's condition, the temperature falling to normal in about a week. A sinus persisted until the child was discharged from hospital six weeks later, but was now healed. Mr. Mayou said that in his first case of this sort, which occurred in 1901, practically the whole of the upper jaw came away as a sequestrum. Nearly all the recorded cases of orbital cellulitis had been due to osteomyelitis of the upper jaw in infants under 1 year old. The route of infection was difficult to understand, as the child rarely had any teeth : he did not believe that it came from the nose, as the ethmoidal cells were not developed. Probably the infection was metastatic, as in osteomyelitis of the long bones.

Mr. Lestre J. PAToN said that he had had a very similar case in a child 4 months old. On making an incision fully an ounce of pus escaped, and a probe detected bare bone. No bone was exfoliated in this case. The sinus eventually closed, leaving remarkably little disfigurement.

Mr. HUMPhREY Neame exhibited a case of Congenital Abnormality

branching remnants of persistent hyaloid vessels. The patient, a female aged $66^{\circ}$ years, had noticed mistiness and "black spots" in the right eye for several years. Vision in the right eve with a correction was 6/36, and in the left $6 / 9$. The right fundus exhibited a number of floating strands in the vitreous arising by a single stalk from the dise. These had no attachment to the lens.

Miss IDa MaNN described briefly the development of the hyaloid artery, showing drawings made from serial sections of the eve of the human fotus at five weeks. The hyaloid artery generally appeared 